\title{
Viscoelasticidad no lineal en asfaltos modificados con polímero
}

\author{
R. Blanco ${ }^{\mathrm{a}^{*}}$, J. Bonilla ${ }^{\mathrm{a}}$ y G. Hernández-Padrón ${ }^{\mathrm{b}^{*}}$ \\ ${ }^{a}$ Departamento de Ing. Química, Instituto Tecnológico de Estudios Superiores de Monterrey, ITESM. \\ Eugenio Garza Sada 2501, C..P. 64849, Monterrey, Nvo. León, Mèxico, \\ *E-mail: blancoricbp@itesm.mx,jbonilla@itesm.mx \\ ${ }^{b}$ Departamento de Nanotecnología, Centro de Física Aplicada y Tecnología Avanzada, Universidad Nacional Autónoma de \\ México, Juriquilla, Querétaro, C.P. 76230, Querétaro, México. \\ E-mail: genoveva@fata.unam.mx
}

Recibido 04-septiembre-2013, Aceptado 10-octubre-2013

\section{Resumen}

El diseño y la construcción de carreteras y autopistas, así como materiales para impermeabilizar que tengan una vida útil más larga, es una de las prioridades de los ingenieros civiles. La selección de materiales adecuados con respecto a las condiciones climáticas y la frecuencia de la carga es la prioridad de los ingenieros químicos. El uso de los Asfaltos Modificados con Polímeros (AMPs) para estas aplicaciones, ha ido continuamente en aumento en todo el mundo, debido a que con una cantidad relativamente pequeña de polímero añadido, puede mejorar significativamente el rendimiento y durabilidad. En la práctica, la reología es una de las herramientas más útiles para el estudio de los AMPs. En particular, la reología no lineal puede dar una información única que ayuda a interpretar cómo el polímero se organiza íntimamente cuando se mezcla con el asfalto. En el pasado, muchos trabajos han descrito el comportamiento lineal de los AMPs. En este trabajo se presenta una breve descripción de las propiedades reológicas lineales. Sin embargo, la atención particular se ha puesto en obtener las propiedades reológicas no lineales tales como viscosidad de cizallamiento $\eta(\dot{\gamma}) \circ \eta(\dot{\gamma}, \mathrm{t})$, a partir de las propiedades lineales medibles utilizando el modelo de Wagner-Lodge ${ }^{[1-2]}$. La región viscoelástica no lineal se describe a través de una función $\Phi(\mathrm{t}, \dot{\gamma})$ combinatoria memoriaamortiguamiento que depende del tiempo ty la deformación ó la velocidad de deformación $\gamma$.

Palabras clave: Modelo de Wagner, reología no lineal, Asfalto Modificado con Polímero

\begin{abstract}
The design and construction of highway roads and waterproofing roof materials with longer service lives is a priority of civil engineers. The selection of appropriate materials with respect to climatic and loading conditions is the priority of chemical engineers. The use of Polymer Modified Asphalts (PMAs) for these applications have been continually increasing worldwide, because a relatively small quantity of added polymer can significantly enhance performance and durability. In practice, rheology is one of the most useful tools for the study of PMAs. Particularly, nonlinear rheology may give unique information that helps to interpret how the polymer organizes when it is blended with asphalt. In the past many papers have described the linear behavior of PMAs. In this paper a brief description of linear PMAs rheological properties and modeling are showed. However a particular attention is paid to rheological properties such as shear viscosity $\eta(\dot{\gamma}), \eta(\dot{\gamma}, \mathrm{t})$ in the nonlinear viscoelastic region using the Wagner model which is a modification of the Lodge model. The nonlinear viscoelastic region is described through a time and strain dependent memory-damping function $\mathrm{F}(\mathrm{t}, \gamma)$.
\end{abstract}

Key words: Wagner model, non linear asphalt rheology, polymer modified asphalts

\section{Introducción}

Una gran cantidad de trabajos en este campo, han sido publicados con relación a la viscoelasticidad lineal para la predicción de propiedades reológicas, junto con la relación que se tiene con la microestructura, lo cual es posible a bajas velocidades de corte y bajas deformaciones ó bajas velocidades de deformación. En la mayoría de los casos los datos fueron obtenidos por medio de mediciones oscilatorias dinámicas a diversas temperaturas y a diferentes frecuencias utilizando un reómetro de geometría de placas paralelas ó cono y plato. Sin embargo no siempre cuando extendemos el estudio a grandes deformaciones es posible encontrar correlaciones entre la estructura y las propiedades debido a que las cadenas de polímero se someten a cambios y configuraciones espaciales complejas cuando están en el asfalto. Es en esta situación cuando la viscoelasticidad no lineal (reología no lineal) emerge como una poderosa herramienta de análisis. En este sentido, la viscosidad de corte y el módulo de relajación son probablemente las funciones no lineales más sencillas que pueden revelar el comportamiento de los AMPs.

\section{Parte Experimental}

Se midieron y modelaron propiedades reológicas lineales y no lineales de asfaltos modificados con copolímero termoplástico de estireno-butadieno- 
estireno (SBS). En todos los casos, las materias primas eran muestras comerciales. El SBS usado es un material en bloque de alto peso molecular (400.000 aprox.) de la Cía. DYNASOL cuya nomenclatura es: SBS-411 ${ }^{\mathrm{MR}}$. Por otra parte, el Asfalto era una muestra comercial no oxidada $($ Pen $=70 \mathrm{dmm})$ de PEMEX AC-20 ${ }^{\mathrm{MR}}$. Las materias primas se caracterizaron antes de hacer la mezcla. El estudio se realizó dentro de una amplia gama de composiciones entre $0 \%$ y $14 \%$. El objetivo principal de este trabajo fue calcular propiedades como $\eta(\dot{\gamma})$, $\eta(\dot{\gamma}, \mathbf{t})$ en la región viscoelástica no lineal (rvnl) a partir de las propiedades de la región viscoelástica lineal (rvl) tales como los módulos dinámicos $G^{\prime}(\omega)$ y G" $(\omega)$, que se realizaron a diferentes temperaturas entre 40 a $180^{\circ} \mathrm{C}$.

\section{Modelo matemático}

Se utilizó el modelo Wagner ${ }^{[1]}$ que es una modificación del modelo de Lodge $^{[2]}$ en la región viscoelástica no lineal, el cual se describe a través de una función de memoria combinada con una función de amortiguamiento $\Phi(\mathrm{t}, \gamma)=\mu(\mathrm{t}) \mathrm{h}(\gamma)$, con ello se calculan funciones como:

$\eta(\dot{\gamma}), \eta(\dot{\gamma}, \mathrm{t})$ ó $\mathrm{N}_{1}(\dot{\gamma}, \mathrm{t})$.

\section{Resultados y discusión}

En las siguientes gráficas se muestra el análisis de datos de AMPs seleccionados: 0\% de SBS (Asfalto puro), 4\% SBS, $8 \%$ SBS y $14 \%$ SBS de acuerdo al modelo de Wagner. En la Figura 1 se muestran las gráficas de viscosidad de corte función de la velocidad de deformación experimental (utilizando la regla de CoxMerz para calcular los datos de $\eta(\dot{\gamma})$ por medio $\eta^{*}(\omega)$ experimentales y se comparan con $(\dot{\gamma})$. Todas las mezclas se midieron a $40^{\circ} \mathrm{C}$.

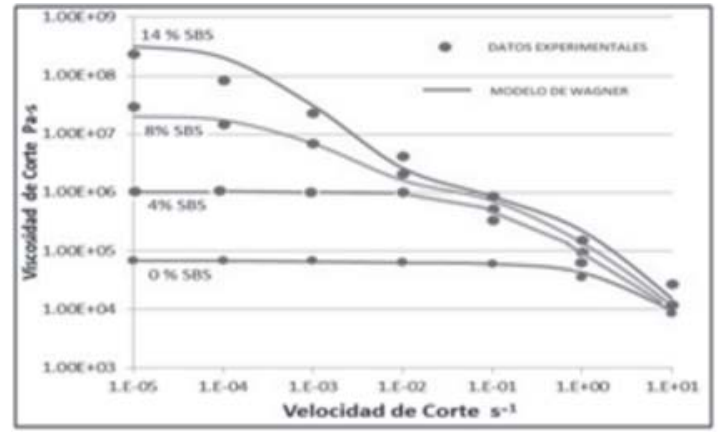

Figura 1. Viscosidad de corte en estado estable $\eta(\dot{\gamma})$ a diferentes velocidades de corte $\dot{\gamma}$ para las mezclas indicadas a 40 ${ }^{\circ} \mathrm{C}$.

Esta gráfica ilustra claramente la influencia de la concentración al 8 y $14 \%$ en el comportamiento de la viscosidad, se notan 2 descensos bruscos en la propiedad viscoelástica, primero al disminuir ( con valor de velocidad de corte de $8 \times 10^{-5} \mathrm{~s}^{-1}$ ) de $2 \times 10^{8} \mathrm{~Pa}$-s a $1.8 \times 10^{6}$
Pa-s y luego continua con un pequeña meseta entre esta valor y $1.5 \times 10^{6}$, la segunda transición aparece alrededor de $1.0 \times 10^{6} \mathrm{~Pa}$-s hasta una zona última de $1.4 \times 10^{4}$. La siguiente curva la de $8 \%$ se parece a la primera pero la caída de la viscosidad aparece aproximadamente en $\dot{\gamma}=$ $1 \times 10^{-4} \mathrm{~s}^{-1}$ con comportamiento subsecuente similar a la primera, estas dos curvas muestran un comportamiento de meseta de hule (como era de esperarse por el dominio en las propiedades del SBS sobre el Asfalto). La tercera curva de $4 \%$ de SBS los valores de la viscosidad son más constantes, con cambio brusco de descenso pero con valores de $=1 \times 10^{-1} \mathrm{~s}^{-1}$ indicando que el sistema tiende a ser newtoniano. Finalmente la curva ultima denota un comportamiento clásico newtoniano, lo que también es de esperarse ya que se trata de asfalto puro ( $0 \% \mathrm{SBS})$, estos comportamientos son similares a los descritos en referencias de vanguardia en este tema ${ }^{[6-8]}$

Así mismo se muestra la viscosidad a $60^{\circ} \mathrm{C}$ como función del tiempo y la velocidad de corte $\eta(\mathbf{t}, \dot{\gamma})$ de las mezclas a 0 y $14 \%$ de SBS. Las estimaciones están ilustradas en las Figuras 2 y 3 , respectivamente.

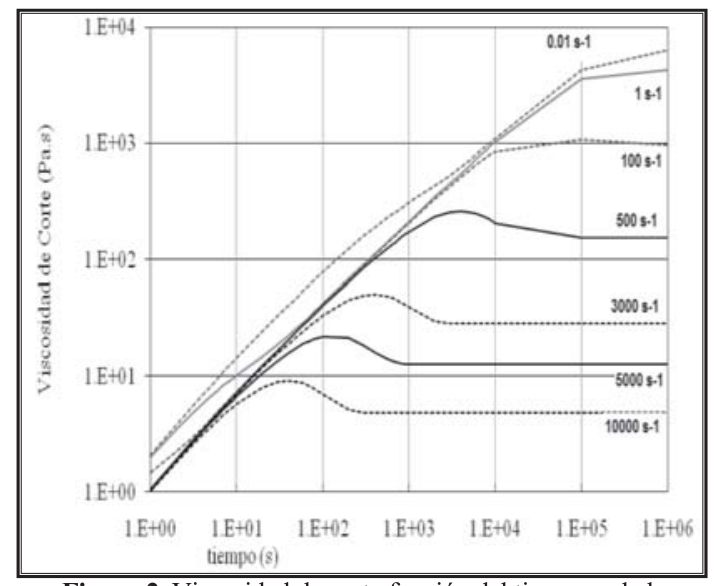

Figura 2. Viscosidad de corte función del tiempo y de la velocidad de corte $\eta(\mathbf{t}, \dot{\gamma})$ para la mezcla al $0 \%$ de SBS a $60^{\circ} \mathrm{C}$

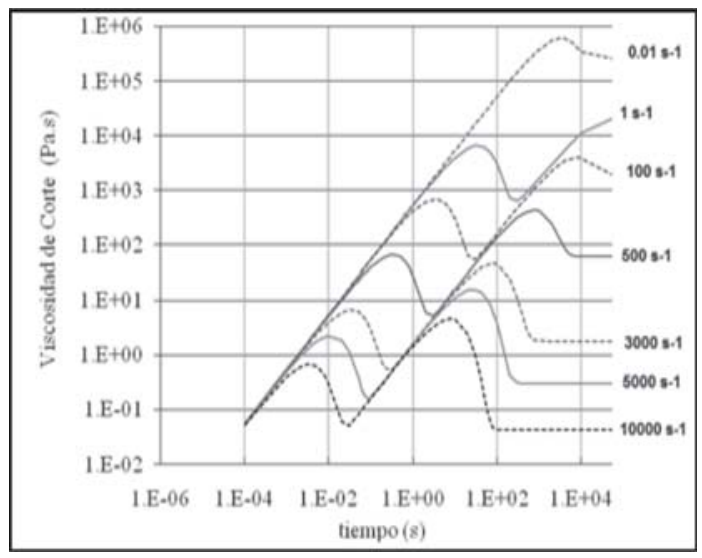

Figura 3. Viscosidad de corte función del tiempo y de la velocidad de corte $\eta(\mathbf{t}, \dot{\gamma})$ para la mezcla al $14 \%$ de SBS a $60^{\circ} \mathrm{C}$ 
El comportamiento de estas gráficas también muestran patrones característicos entre un sistema no newtoniano y el newtoniano, por el comportamiento bimodal mostrado conforme va cambiando el tiempo.

\section{Conclusiones}

Es bien sabido que todos los expertos en reología desean correlacionar la estructura del material a nivel molecular con sus propiedades macroscópicas, en este caso visco-elásticas. El haber usado un modelo fenomenológico, que se basa únicamente en parámetros físicos medibles, permitió obtener propiedades reológicas no lineales a partir de propiedades lineales, factor clave para inferir la microestructura de los AMPs, proporcionando así una herramienta predictiva. Los investigadores que trabajan con este sistema tienen todavía un largo camino por recorrer. Los asfaltos modificados con polímeros son mezclas de estos dos materiales y el estudio y la comprensión son muy importantes para la predicción de la vida útil en los servicios. Cuando la mezcla es una red polimérica donde se hincha debido a los componentes del asfalto, pero mantiene las principales características de su arquitectura original, las propiedades reológicas de los AMPs son básicamente las del polímero. Durante las últimas décadas, la atención se ha prestado principalmente a las propiedades lineales de estos materiales. Sin embargo, las propiedades no lineales son prometedores y por medio de ellas revelar los "secretos" de estos materiales.Fue posible calcular las propiedades reologicas no lineales como $\eta(\dot{\gamma}), \eta(\dot{\gamma}$ ,t) a partir de propiedades lineales G'( $\omega)$ y G”'( $\omega)$.

\section{Referencias}

1. Wagner MH. Analysis of time-dependent non-linear stress growth data for shear and elongational flow of a lowdensity branched polyethylene melt. Rheol Acta 1976; 15:136-42.

2. Lodge AS. Elastic liquids. London UK: Academic Press; 1964. p. 389.

3. Bonilla,J. Wagner Modeling application on Polypropylene Resins, 1996, PHD Thesis, Instituto Tecnológico de Monterrey

4. Laun HM. Description of the non-linear shear behavior of a low densitypolyethylene melt by means of an experimentally determined strain dependent memory function. Rheol Acta 1978; 171:1-15.

5. Ferry, "Viscoelastic Properties of Polymers "J John Wiley \& Sons, Inc, 1970

6. Polacco G, Stastna J, Biondi D, Zanzotto, Relation between polymer architecture and nonlinear viscoelastic behavior of modified asphalts, Colloid and Interface Science, Elsiever 2006, 230-245

7. Polacco G, Stastna J, Biondi D, Vlachovicova Z, Zanzotto P. Time scales in polymer modified asphalts. Macromol Symp 2005; 228:41- 50 .

8. 8. Vlachovicova Z, Stastna J, MacLeod D, Zanzotto L. Shear deformation and material properties of polymermodified asphalt. Pet Coal 2005; 47:38-48. 